\title{
DISTINTA VALORACIÓN DE LOS AGUACEROS EN EL TIEMPO: LA ALARMA DE NOVIEMBRE DE 1984 EN ALICANTE
}

\section{Margarita Box Amoros}

\section{CONDICIONES METEOROLÓGICAS Y AGUACEROS EN NOVIEMBRE DE 1984}

Durante los días 9, 10 y 11 de noviembre de 1984,las condiciones meteorológicas imperantes en el SE. peninsular, quedaban definidas por una situación sinóptica habitual para la época del año. Se trataba de la formalización en altura de un proceso de gota fría y si incuestionables son las importantes consecuencias pluviométricas que se pueden derivar del mismo $^{1}$, resulta también imprescindible valorar este fenómeno en su justa medida, puesto que no es el único responsable de aguaceros torrenciales, ni tan sólo su exclusiva presencia es suficiente para desencadenar precipitaciones de cierta entidad ya que para que ello se produzca es necesario el concurso de otra serie de factores de capital importancia en el proceso.

\section{La percepción de las consecuencias meteorológicas}

Dejando aparte cuestiones de estricta índole climática que se analizarán posteriormente, un hecho que resalta, en una primera aproximación al tema, es el de la distinta percepción del fenómeno por el hombre a lo largo del tiempo. Resulta, por tanto, interesante hacer, en principio, una valoración histórica del mismo que permita comprender cómo un mecanismo que ha existido siempre en nuestro ámbito, las lluvias torrenciales, ha tenido una significación radicalmente distinta a la que hoy posee. [52]

Efectivamente, la dinámica atmosférica que rige la climatología de nuestra zona, se caracteriza desde el punto de vista de las precipitaciones por la presencia de unos pocos chubascos de fuerte intensidad horaria que son los que proporcionan la casi totalidad del volumen pluviométrico anual. Este no es un hecho nuevo sino que, tradicionalmente, el hombre de nuestros campos y ciudades ha tenido constancia de ello, intentando adaptar sus modos de vida y su economía a un medio que presentaba estas características físicas. Baste para comprobar esta afirmación, con acudir a la obra de un clásico de la literatura geográfica, la del insigne botánico Cavanilles que, en su recorrido por las tierras del antiguo Reino de Valencia a fines del s. XVIII, relató los esfuerzos del campesinado por hacer productivo un medio donde la escasez de agua constituía el principal problema de la agricultura, de manera que su objetivo se encaminó siempre a poder aprovechar al máximo las aguas de lluvia, aún y cuando éstas se produjeran de manera torrencial. Resulta, en este sentido, significativo, el párrafo de su obra dedicado al término de Agost y en el que se afirma lo siguiente:

\footnotetext{
«Quien ignore se suma la escasez de agua en parte del reyno, y que á veces un solo riego basta para asegurar y aumentar las cosechas, extrañará ver salir los labradores hácia sus haciendas quando empieza á tronar, ó amenaza alguna tempestad: los truenos, que en otras partes del reyno sirven de señal para retirarse á sus habitaciones lo son aqui para desampararlas y salir en busca de las aguas y deseado riego: se fecundan entónces los olivos, higueras, almendros, viñas y algarrobos; y el suelo entero se mejora con el cieno que traen las aguas» ${ }^{2}$.
}

Es este texto sumamente sugestivo puesto que de su análisis se puede extraer la conclusión de que esas precipitaciones copiosas no constituían para el agricultor tradicional un motivo de preocupación y temor, al contrario, eran el elemento principal de su economía, puesto que como afirma Cavanilles, un solo riego era suficiente para proporcionarles buenas cosechas, lógicamente de ello se deduce que estas lluvias debían ser abundantes. Ante ellas, el agricultor 
desconocía el miedo y se lanzaba a sus campos para abrir acequias y partidores con el fin de [53] que las aguas de lluvia penetraran en ellos. Situación bien distinta a la de los días 9, 10 y 11 de noviembre de 1984, donde la ciudad quedó prácticamente desierta ante los partes meteorológicos ofrecidos por los medios de comunicación. Eran unas lluvias para este campesino que le iban a asegurar la cosecha de ese año y además, iban a fertilizar el suelo por el légano arrastrado con las aguas de avenida.

De todo lo anteriormente expuesto, se trasluce que este tipo de lluvias, por ser casi las únicas aportaciones de agua a nuestros campos, eran para los agricultores como una especie de bendición y, en función de ello, construyeron sus sistemas de regadío de boqueras, es decir, la instalación de una presa o partidor en el cauce de los barrancos, de forma transversal a los mismos que, en los momentos de avenida, desviaba las aguas por medio de canales hasta los terrazgos ${ }^{3}$.

Igualmente, el aprovechamiento de las aguas de turbias es bien patente en el cauce del Río Montnegre o Seco donde ya desde antes de la construcción del Pantano de Tibi, en el s. XVI, existían unas primitivas presas sobre las que con posterioridad se edificarían los azudes de Muchamiel y San Juan y más recientemente el de Campello ${ }^{4}$ a partir de los cuales y mediante un complicado sistemas de acequias se regaba la Huerta de Alicante. Estas aguas de avenida, incrementaban el caudal normal de riego y por ello, las fuertes precipitaciones estaban consideradas como muy beneficiosas al poder disponer los agricultores de unos volúmenes complementarios, aunque por supuesto, en ocasiones, las precipitaciones torrenciales fueran la causa de la destrucción de los campos y de las infraestructuras viarias.

En definitiva, el campesino tradicional conocía muy bien el medio en el que se asentaba y tenía una percepción del mismo que para nada suponía una visión peyorativa sino que las lluvias otoñales eran esperadas con verdadera ansiedad.

La situación es hoy bien distinta, el paso de una economía tradicional «cuasi» de subsistencia a un sistema donde el factor principal es la rentabilidad, ha determinado una variación en los sistemas de cultivo y [54] regadío. Las parcelas ya no son esas estrechas terrazas que jalonaban las vertientes de nuestras montañas sino amplias unidades de explotación para cuyo riego ya no sirve la eventualidad de unas precipitaciones esporádicas, sino que lo que se pretende es disponer de unos recursos hídricos a lo largo de todo el año y ello se hace explotando las reservas del subsuelo, o trayendo este agua desde lejanos lugares (Trasvase Tajo-Segura, Riegos de Levante y utilización de aguas depuradas). Con ello, el sistema de boqueras ha dejado de ser funcional, y las aguas de avenida ya no se desvían sino que prácticamente todos los volúmenes circulan por los lechos de las ramblas hasta su desembocadura. Además, el hombre por sus necesidades de expansión ha modificado los cauces de las ramblas, hasta el punto de que algunos han desaparecido de la propia topografía, o incluso, se ha asentado en ellos, construyendo en los conos de deyección sus viviendas. Sin embargo, las escorrentías derivadas de las lluvias han tenido siempre sus caminos bien trazados y cuando se producen copiosas precipitaciones, las aguas recobran sus antiguos lechos y es, precisamente, en este factor en el que hay que buscar la peligrosidad de unos aguaceros torrenciales y donde reside el temor de ese hombre que es hoy un ser eminentemente urbano ${ }^{5}$.

Por ello y sin pretender restar significación al tema, resulta necesario que el hombre llegue a estimar el fenómeno en sus justos límites. Hoy, en la calle, son pocas las personas que desconocen el término y oír hablar de «la gota fría» o simplemente de «la gota» resulta algo tan natural como abrumador puesto que durante esos días del mes de noviembre pareció existir una especie de psicosis urbana que, en determinados momentos, pudo llegar a causar auténtico pánico en algunos sectores de la ciudad, bien es verdad que este temor estaba, en cierta manera justificado puesto que la población tenía aún muy recientes las huellas de las catastróficas inundaciones de 1982, derivadas precisamente de este mismo proceso. 
Análisis de la situación de 1984 y su comparación con la de 1982

Para el desencadenamiento de lluvias torrenciales, en el ámbito del SE peninsular, el factor más frecuente es [55] la formalización de un proceso de gota fría en altura, pero este es un elemento a considerar y no el único ya que han de intervenir además otra serie de mecanismos que, indudablemente, no jugaron en noviembre de 1984.

Las condiciones meteorológicas que dominaron la vertiente mediterránea española y, consecuentemente, a la ciudad de Alicante durante los días 9, 10 y 11 de noviembre de 1984, provocaron por sus posibles repercusiones una desazón ciudadana, si es lícito aplicar este término, al ser puestos en estado de alerta la casi totalidad de servicios públicos de emergencia. Indudablemente, las autoridades locales estimaron conveniente tomar el mayor número de precauciones posibles ante los partes ofrecidos por el Servicio Meteorológico Nacional que seguía paso a paso la evolución de la perturbación, si bien es verdad que se cuenta con una red de observatorios reducida y no pudiendo por ello ser efectuadas mediciones y análisis continuados en las distintas capas de la atmósfera y, quizás lo que es más fundamental, la naturaleza y evolución de las masas de aire que ubicadas sobre la superficie marina mediterránea son las auténticas responsables de precipitaciones abundantes. Es éste un factor básico para poder llegar a calibrar con precisión la magnitud del fenómeno.

El análisis de la situación sinóptica en las distintas capas de la atmósfera mostraba de manera contundente la progresiva formalización de un proceso de gota fría en altura ubicado sobre la vertical de la Península Ibérica que podía llegar a producir precipitaciones muy abundantes por la concurrencia en superficie de una baja frontal que centrada en el Mediterráneo era, enormemente, proclive al envío sobre nuestras costas del aire estancado sobre esta superficie marina y, lógicamente, con un alto contenido en vapor de agua.

Con ello se disponía, efectivamente, de los mecanismos propicios para el desencadenamiento de aguaceros torrenciales, hecho éste que sin lugar a dudas motivó la preocupación de los responsables del Servicio Nacional.

Con las diferencias lógicas entre la situación de octubre de 1982 y la de noviembre de 1984, los campos de [56] presiones tanto en altura como en superficie mostraban una distribución muy similar, es decir, presencia en altura de un embolsamiento de aire anormalmente frío, fruto de la trasgresión hacia el sur de una intensa vaguada resultante de la fuerte meandrización del jet y ubicación en superficie de un campo de presiones, en este caso concreto, como ya se ha dicho, de una baja frontal que vehiculaba hacia la costa aire del Mediterráneo. 


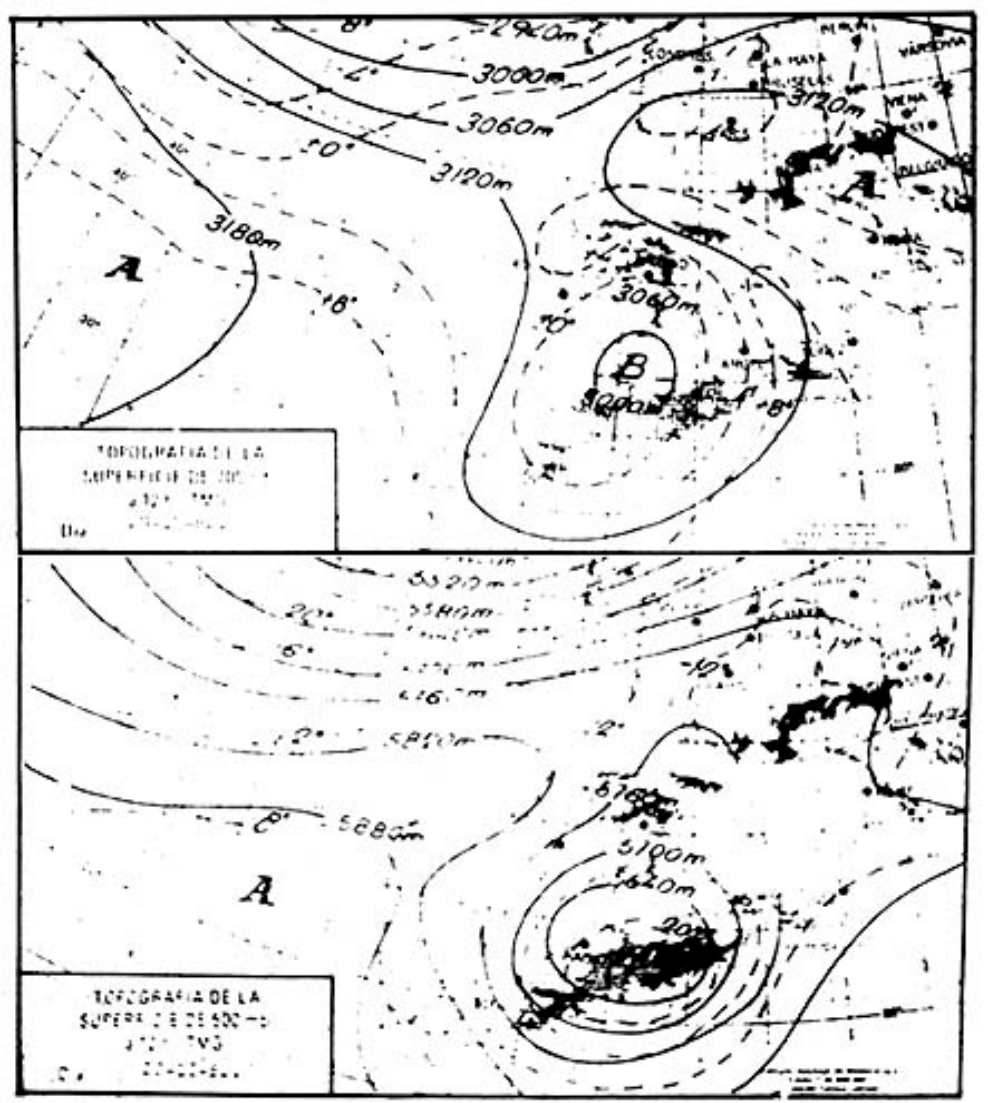

1.- Topografía de $500 \mathrm{mb}$ (20-X-82). Baja desprendida ubicada sobre el norte de África abarcando en su radio de acción a la Península Ibérica. Sobre la vertical de Alicante la isoterma registra $-16^{\circ} \mathrm{C}$.

[57]

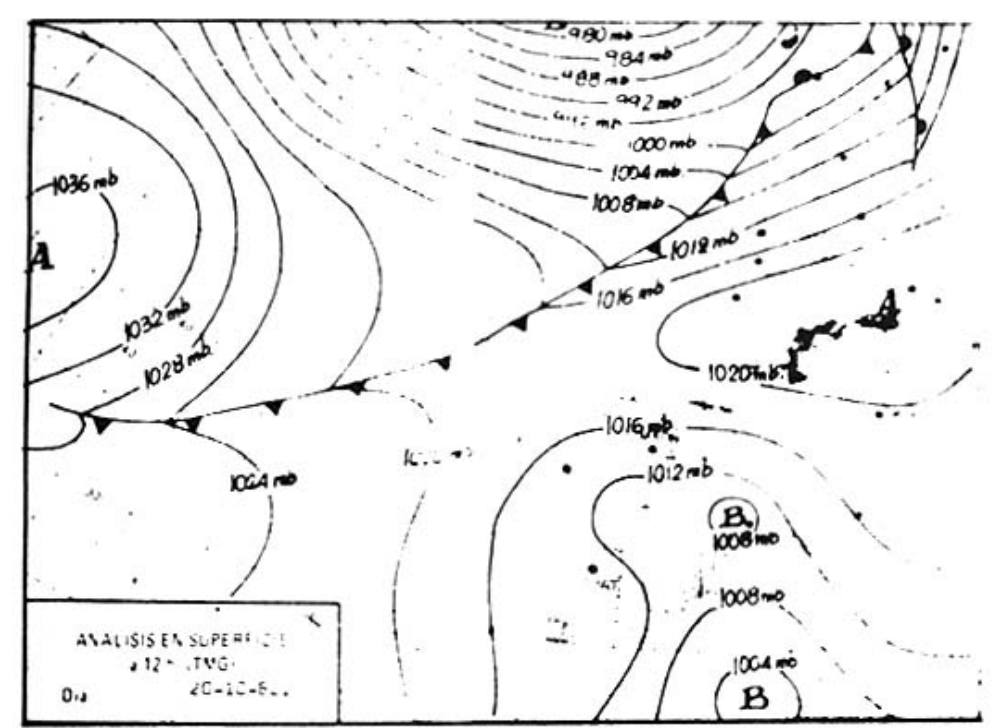

2.- Mapa de superficie (20-X-82). Bajas presiones frente a las costas murciano-almerienses que por su rotación ciclónica envían sobre la fachada del SE peninsular vientos húmedos de componente ESE 


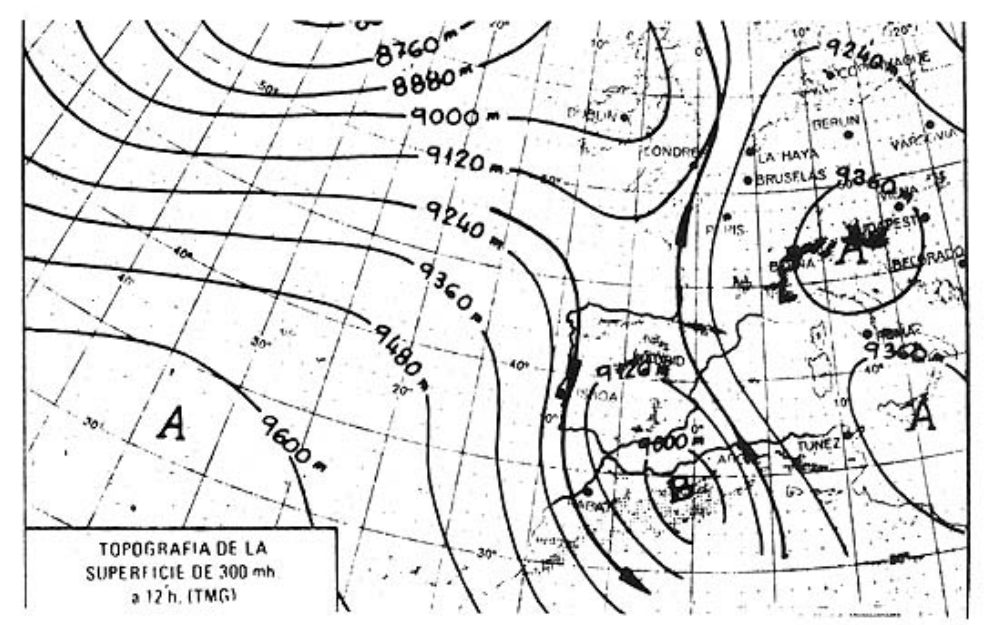

3.- Topografía de $300 \mathrm{mb}$ (10-XI-84). Gota fría centrada en el norte de África y proceso de difluencia en delta sobre el segmento norte valenciano

[58]

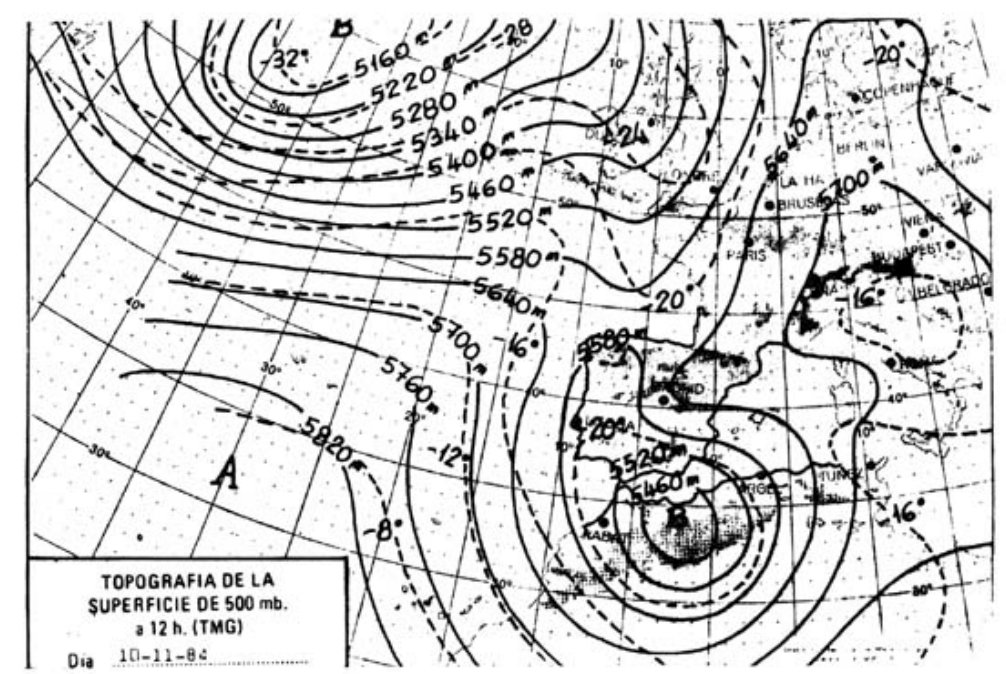

4.- Topografía de $500 \mathrm{mb}$ (10-XI-84). Embolsamiento de aire anormalmente frío, fruto de la trasgresión de una vaguada fría, ubicado sobre el norte de África 


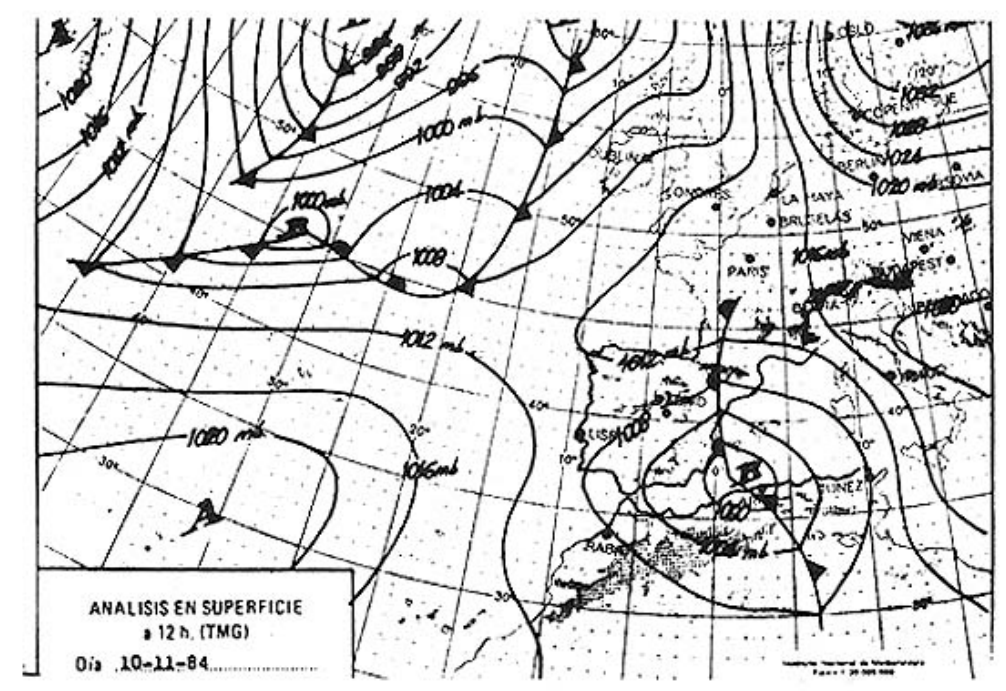

5.- Mapa de superficie (10-XI-84). Baja frontal frente a las costas norteafricanas que vehicula hacia el litoral mediterráneo español, flujo de dirección SE-NW

[59]

Cabría entonces plantearse la cuestión de por qué con situaciones sinópticas parecidas, las consecuencias pluviométricas no fueron iguales a las de octubre de 1982. A este respecto resulta significativo que mientras entre las 0 y las 7 h. del día 20 de octubre de 1982, en el Observatorio de Ciudad-Jardín (Alicante) se recogió un total de $200,7 \mathrm{~mm}^{6}$, es decir algo más del $60 \%$ de la precipitación media anual, en noviembre del 84, se contabilizó tan sólo un volumen de $60,71 / \mathrm{m}^{2}$, teniendo además en cuenta que este total se distribuyó a lo largo de 3 días, con valores de 19,0 mm para el día 9, 27,5 mm para el día 10 y 13,6 $\mathrm{mm}$ para el día $11^{7}$. A priori, es necesario partir de un hecho básico determinado por la propia fecha, es decir, la formalización de la perturbación en el mes de noviembre. Efectivamente aún y cuando es la época otoñal la que registra el mayor volumen de precipitaciones en nuestro ámbito, éstas son el resultado de unos pocos aguaceros que desencadenan lluvias torrenciales y, en algún caso, inundaciones, siendo el mes de octubre el que a nivel estadístico registra el mayor número de chubascos de intensidades superiores a los $50 \mathrm{~mm} / 24 \mathrm{~h}$, mientras que el mes de noviembre, acoge un porcentaje mínimo de este tipo de precipitaciones. La causa de ello estriba en una menor temperatura de las aguas marinas puesto que, a pesar de la inercia térmica, se ha producido ya una pérdida calorífica experimentada en el transcurso de los dos primeros meses otoñales. Este hecho resulta trascendental puesto que el aire estancado sobre esa superficie marina tiene, en consecuencia, una menor riqueza higrométrica, que, en definitiva, es el elemento indispensable para la caída de precipitaciones tras el proceso de condensación. En resumen y como primera premisa a considerar, hay que contar con unas masas de aire con un contenido en vapor de agua mucho más reducido que en los meses de septiembre y octubre.

La cuantificación de este dato, viene dada a partir de la tensión de vapor; es por ello de gran interés conocer los valores de la misma durante esos días y, lógicamente, a efectos de contraste su comparación con los de octubre de 1982. Así mientras que a partir de las $18 \mathrm{~h}$. del día 19 de octubre de 1982, las tensiones de vapor registradas en el Observatorio Meteorológico de Ciudad Jardín experimentaron un incremento progresivo pasando de 11,2 mb a [60] 16,5 mb hecho indicativo de un constante enriquecimiento higrométrico de las masas de aire ${ }^{8}$, en noviembre de 1984, las tensiones de vapor durante los días 9, 10 y 11 se mantuvieron estables y prácticamente constante en torno a los $11 \mathrm{mb}$, tal y como se desprende del siguiente cuadro: 


\begin{tabular}{|c|c|c|c|}
\hline$\underline{\text { Horas }}$ & 9-XI-84 & $\underline{10-X I-84}$ & $\underline{11-X I-84}$ \\
\hline 7 & 12,3 & 10,2 & 11,2 \\
\hline 13 & 9,4 & 11,3 & 12,4 \\
\hline 18 & 11,4 & 11,3 & 11,2 \\
\hline
\end{tabular}

No había, en consecuencia, aumento del contenido en vapor de agua, ni tan siquiera sus valores se aproximaban a los de octubre de 1982.

Íntimamente vinculado con el contenido en vapor de agua de las masas de aire se encuentra el llamado punto de rocío. Existió, en efecto, una menor proximidad de las masas de aire en noviembre de 1984 a alcanzar su punto de rocío, es decir a quedar saturadas ya que mientras en octubre las diferencias entre temperatura y punto de rocío llegaron a ser de tan sólo $0,3^{\circ} \mathrm{C}$, en noviembre estos valores se alejaron siendo siempre superiores a $0,8^{\circ} \mathrm{C}$, alcanzando, incluso diferencias de hasta $8,4^{\circ} \mathrm{C}$ el día 9 a las $13 \mathrm{~h}$.

Este hecho resulta también muy importante puesto que mientras en octubre las masas de aire evolucionaron pseudo-adiabáticamente desde niveles muy bajos, en torno a los 150-200 m, en esta ocasión el proceso adiabático húmedo se operó por término medio a mucha mayor altura, de manera que la ganancia térmica respecto al aire envolvente se vio notablemente disminuida, frenándose así el fenómeno de ascenso. A ello contribuyó también los valores del gradiente térmico estático en la vertical que llegaron a ser, incluso, inferiores a $0,5^{\circ} \mathrm{C} / 100 \mathrm{~m}$ en los primeros $1.500 \mathrm{~m}$, con lo que el aire ascendente perdía frente al envolvente de una manera rápida su ventaja térmica inicial y si esta es la clave de cualquier ascenso convectivo, en noviembre de 1984, este factor jugó un papel de freno ante una elevación prácticamente sin límites, como ocurrió en octubre del 82 y que fue capaz de generar copiosas lluvias. [61]

Finalmente, fue el menor potencial higrométrico el que jugó un papel capital en la no elaboración de unas precipitaciones copiosas e intensas, hecho éste que en definitiva, es el mecanismo generador de inundaciones por la acumulación de unos totales pluviométricos en un corto espacio de tiempo.

Sin embargo, no podemos dejar a un lado la distribución irregular del fenómeno porque, efectivamente, abundantes lluvias se produjeron durante estos días del mes de noviembre aunque en otros lugares de nuestra geografía, con lo que queda demostrado que si bien el escaso contenido en vapor de agua fue un elemento importante, en otras zonas (como por ejemplo, en la provincia de Albacete), donde se recogieron $66 \mathrm{~mm}$ el día 10-XI-84, intervinieron factores diferentes que potenciaron el fenómeno. Por ello, lo absolutamente necesario es una adecuada red de observatorios, de manera que una vez iniciado el desarrollo de las nubes convectivas, se puedan efectuar estimaciones aproximadas de las cantidades de precipitación y con ello prevenir las posibles consecuencias negativas de unos chubascos abundantes.

\section{NOTAS}

1 Vid. Rev. Estudios Geográficos n ${ }^{\circ}$ 170-171 C.S.I.C. Madrid, 1983 (Número monográfico sobre las inundaciones en el Pirineo Central, Cataluña, Valencia y Murcia en octubre-noviembre de 1982).

2 Cavanilles, A. J.: Observaciones sobre la historia natural, geografía, agricultura, población y frutos del Reyno de Valencia. Imprenta Real. Madrid, 1797 (edic. Facsímil) Valencia, 1981 p. 524.

3 MORALES GIL, A.: «El riego con aguas de avenida en las laderas subáridas» Papeles del Departamento de Geografía Universidad de Murcia, Curso 1968-69 p. 170 y ss. 
4 ALBEROLA ROMA, A.: El pantano de Tibi y el sistema de riegos en la Huerta de Alicante Inst. Juan Gil Albert. Alicante, 1984 pp. 70-79.

5 Vid. MORALES GIL, A., BRU RONDA, C. y BOX AMOROS, M.: «Las crecidas en los barrancos de las Ovejas y Agua Amarga, Alicante. Octubre-1982» en Estudios Geográficos Op. cit. p. 143-170 y RAMOS HIDALGO, A.: «Los mecanismos y los procesos de inundación en la ciudad de Alicante» en Lluvias torrenciales e inundaciones en Alicante Op. cit. p. 73-98.

6 GIL OLCINA, A.: «Lluvias excepcionales en la noche del 19 al 20 de octubre de 1982 y riada en el barranco de las Ovejas» en Lluvias torrenciales e inundaciones en Alicante Op. cit. p. 7. [62]

7 Los datos climáticos referidos a los días 9-10 y 11 de noviembre de 1984 han sido obtenidos en el Observatorio Meteorológico de Ciudad-Jardín (Alicante).

8 GIL OLCINA, A. «Lluvias excepcionales...» Op. cit. p. 6. 